\title{
Mechanical and Control Design of an Industrial Exoskeleton for Advanced Human Empowering in Heavy Parts Manipulation Tasks
}

\author{
Alessandro Mauri ${ }^{1,2}{ }^{\mathbb{D}}$, Jacopo Lettori ${ }^{1,3}{ }^{10}$, Giovanni Fusi ${ }^{4}$, Davide Fausti ${ }^{4}$, Maurizio Mor ${ }^{4}$, \\ Francesco Braghin ${ }^{2}$, Giovanni Legnani ${ }^{1,3}$ and Loris Roveda $1,5, *$ (i) \\ 1 Consiglio Nazionale delle Ricerche (CNR), Istituto di Sistemi e Tecnologie Industriali per il Manifatturiero \\ Avanzato (STIIMA), 20133 Milano, Italy; alessandro7.mauri@mail.polimi.it (A.M.); \\ j.lettori@studenti.unibs.it (J.L.); giovanni.legnani@unibs.it (G.L.) \\ 2 Department of Mechanical Engineering, Politecnico di Milano, 20156 Milano, Italy; \\ francesco.braghin@polimi.it \\ 3 Department of Mechanical and Industrial Engineering, University of Brescia, 25121 Brescia, Italy \\ 4 Polibrixia, 25123 Brescia, Italy; giovanni.fusi@polibrixia.it (G.F.); davide.fausti@polibrixia.it (D.F.); \\ maurizio.mor@polibrixia.it (M.M.) \\ 5 Istituto Dalle Molle di Studi sull'Intelligenza Artificiale (IDSIA), Scuola Universitaria Professionale della \\ Svizzera Italiana (SUPSI), Università della Svizzera Italiana (USI), IDSIA-SUPSI, 6928 Manno, Switzerland \\ * Correspondence: loris.roveda@idsia.ch
}

Received: 4 May 2019; Accepted: 31 July 2019; Published: 2 August 2019

\begin{abstract}
Exoskeleton robots are a rising technology in industrial contexts to assist humans in onerous applications. Mechanical and control design solutions are intensively investigated to achieve a high performance human-robot collaboration (e.g., transparency, ergonomics, safety, etc.). However, the most of the investigated solutions involve high-cost hardware, complex design solutions and standard actuation. Moreover, state-of-the-art empowering controllers do not allow for online assistance regulation and do not embed advanced safety rules. In the presented work, an industrial exoskeleton with high payload ratio for lifting and transportation of heavy parts is proposed. A low-cost mechanical design solution is described, exploiting compliant actuation at the shoulder joint to increase safety in human-robot cooperation. A hierarchic model-based controller with embedded safety rules is then proposed (including the modeling of the compliant actuator) to actively assist the human while executing the task. An inner optimal controller is proposed for trajectory tracking, while an outer safety-based fuzzy logic controller is proposed to online deform the task trajectory on the basis of the human's intention of motion. A gain scheduler is also designed to calculate the inner optimal control gains on the basis of the performed trajectory. Simulations have been performed in order to validate the performance of the proposed device, showing promising results. The prototype is under realization.
\end{abstract}

Keywords: industrial exoskeleton design; industrial exoskeleton control; human-robot collaboration; optimal control; empowering fuzzy control

\section{Introduction}

Exoskeletons are one of the key technologies to assist humans in a wide range of applications, such as rehabilitation, daily activities and so forth [1-3]. In particular, the adoption of exoskeletons in industrial applications is nowadays a hot-topic [4], since their capabilities to assist humans executing onerous tasks $[5,6]$. 


\subsection{Industrial Exoskeletons Design Solutions}

Industrial exoskeletons can be classified as passive and active. Passive solutions are not provided by actuation, indeed, they use springs and/or dampers to store energy from human's motion and releasing it when required [7,8]. Passive exoskeletons are commonly supporting the human operator in order to relieve him/her from repetitive tasks, while improving ergonomics [9]. Considering upper limbs solutions, different devices are available on the market [10-13]. Such exoskeletons assist humans in specific tasks, e.g., in over-the-head tasks, supporting the arm to reduce muscular stress. The main advantages of these solutions are the reduced weight and size, do not requiring motors and batteries. However, the comfortable range of postures for the human worker is restricted to specific configurations (such as in the over-the-head tasks assistance), making passive exoskeleton typically tailored to specific applications. Proposed solutions also consider a limited payload, since active assistance cannot be generated. In addition, common solutions does not support the forearm, therefore not guaranteeing the elbow support in case, e.g., of heavy parts transportation.

Active exoskeletons [14] are instead provided by actuation, allowing to empower the human worker. Different solutions have been developed in order to face different tasks, adopting different kinematics and hardware solutions [15-17]. Many solutions are available on the market, facing different tasks and scenarios. The Panasonic Corporation has developed an active device, called AWN03, that supports the operator's back when lifting heavy loads [18] thanks to electric motors. Another active human's back support has been developed in Reference [19]. AUTON proposes a back-exoskeleton to support workers in the transportation of heavy parts [20]. Muscle Suit by Innophys is an active back-support for lifting tasks [21]. HAL exoskeleton developed by Tsukaba University [22] is a full body exoskeleton for medical and industrial applications. Sarcos Robotics proposes the full-body exoskeleton Guardian XO for heavy tasks execution [23]. The proposed state-of-the-art solutions are still high-cost, characterized by a complex design, having a limited payload ratio (i.e., the ratio between the exoskeleton payload and the weight of the device) and not involving compliant actuation. Compliant actuation, such as series elastic actuation and variable stiffness actuation [24-26] are increasingly implemented in human-robot interaction tasks [27-29]. A compliant actuation, in fact, can intrinsically increase the safety in human-robot collaboration at the hardware level, having a deformable structure. Some upper limbs exoskeletons exploiting compliant actuation can be found for medical/rehabilitation applications [30,31]. However, due to the higher target payloads and increased design and implementation complexity, available state-of-the-art active industrial upper limbs exoskeletons are not equipped with compliant actuation. The safety issues are therefore tackled only at the software side (i.e., saturating velocities/forces). In addition, low-cost solutions can only be found in rehabilitation/medical domain, where limited power is required [32,33].

\subsection{Exoskeletons Control Solutions}

Exoskeleton control is widely investigated in order to assist humans in different applications [34]. Many control approaches have been developed, integrating different sensors and control techniques. Brain-control schemes have been developed exploiting a electroencephalogram signals [35]. Surface electromyograpy measurements have been exploited in order to control the exoskeleton on the basis of the human's muscles activation [36,37], also exploiting variable impedance control [38]. Admittance force control has been also exploited in order to control the exoskeleton on the basis of the measured interaction between the human and the robot [39]. External devices and measurement systems have also been used to control the exoskeleton on the basis of muscular activation, such as the Myo armband [40] or IMU sensors [41]. Common state-of-the-art approaches, however, show difficulties in the estimation of the human intention, especially while manipulating (partially) unknown payloads. Moreover, common approaches does not allow to online regulate the assistance given to the human during the task on the basis of the human-robot interaction. In addition, safety is commonly tackled in the controller only as a pre-defined saturation on the control action. Considering the empowering scenario and the manipulation of a (heavy) part, safety-based rules modulating the assistance to the 
human on the basis of the current interaction state (i.e., velocity, interaction force and derivative of the interaction force) should be included in the controller.

\subsection{Paper Contribution}

The aim of this paper is to fill the gap above described in the industrial exoskeleton field. More in details, the paper proposes (i) the mechanical design of a low-cost exoskeleton (hardware costs $<10,000$ Euro) for industrial applications with (ii) high payload ration $(>0.8)$, (iii) involving compliant actuation (to achieve intrinsic safety in human-robot interaction), together with (iv) the design of an empowering safety-based control framework.

A lifting and transportation task of a heavy component has been considered as an objective for the exoskeleton design specifications definition (case study: car bumper part with weight of $10 \mathrm{~kg}$ ). On the basis of such task, the kinematics of the exoskeleton has been defined, together with the performance required by the exoskeleton-objective (i) and (ii). A series elastic actuator (SEA) has been designed for the shoulder joint to embed compliance into the device-objective (iii). The SEA has been designed exploiting a compliant transmission (i.e., a compliant belt) between the shoulder joint motor and the link side. The target belt compliance has been calculated in order to achieve a target equivalent shoulder joint compliance. On the basis of such specifications, components from the market (e.g., motors, elastic belt, etc.) has been selected to implement the designed solution.

The proposed empowering controller has been designed in order to actively assist the human during the task execution. Intrinsic safety rules have been embedded into the control design in order to modulate the assistance on the basis of the current interaction state (i.e., velocity, interaction force and derivative of the interaction force) -objective (iv). Furthermore, the controller has been designed to be robust to (partially) unknown payloads (i.e., the weight of the part). A hierarchic controller has been designed, composed by an inner optimal controller (for trajectory tracking purposes) and by an outer safety-based fuzzy logic controller (for human empowering purposes), online modulating the assistance. The inner model-based controller includes the compliant modeling of the shoulder joint. The outer controller (on the basis of the proposed membership functions) is capable to identify the intention of motion of the human, reacting consequently. Moreover, a gain scheduler has been designed in order to store inner optimal control gains as a function of the performed task trajectory (i.e., control gains are a function of the executed trajectory).

Simulations studies have been performed in order to validate the proposed approach, simulating different task scenarios. Simulation results are promising and the proposed methodology will be applied to the real exoskeleton for final evaluation. The proposed exoskeleton is under realization.

\section{Task Specifications \& Exoskeleton's Design Guidelines}

\subsection{Task Description}

Considering the industrial context, the main purpose of the proposed exoskeleton is to relieve the human from lifting efforts of heavy parts, while actively assisting him/her during the task.

In order to design the proposed exoskeleton, a bumper lifting task in a car assembly line (Figure 1a) has been considered as the reference task. Such task implies a target payload for the upper limbs exoskeleton equal to $P_{e}=10 \mathrm{~kg}$ (i.e., each exoskeleton arm has to lift-in ideal conditions $-P_{a}=5 \mathrm{~kg}$ ).

In the following, the task is analyzed in order to define the exoskeleton configurations assumed during the task execution. Two main configurations will be assumed by the human wearing the exoskeleton during reference task (Figure 1b):

- Configuration 1: The arm is extended along the operator's trunk and the elbow configuration is $90^{\circ}$ flexed;

- Configuration 2: The shoulder is flexed of $90^{\circ}$ and the upper limb is completely extended. This is the most critical configuration considering the required motor torques. 


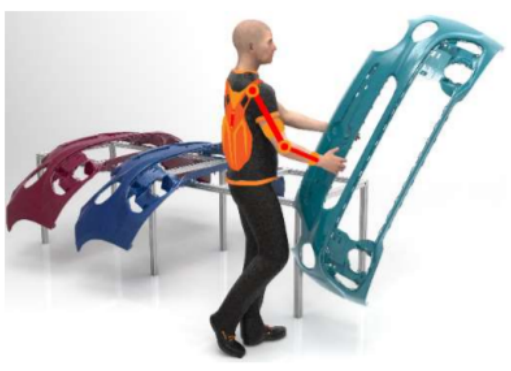

(a)

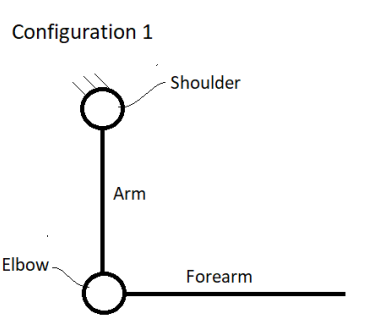

Figure 1. (a) Reference task: car bumper lifting and transportation. (b) Human arm + exoskeleton configurations during task execution.

More in details, the task is composed of nine phases (Figure 2):

- phase 0: the operator configures the upper limbs in configuration 1 and the exoskeleton is turned on;

- $\quad$ phase 1: the operator moves the upper limbs without the external load, in order to grasp the object (critical configuration 2);

- $\quad$ phase 2: the operator grasps the object (critical configuration 2);

- phase 3: the operator moves back to configuration 1 carrying the part;

- $\quad$ phase 4: the operator transports the object maintaining upper limbs in configuration 1;

- phase 5: the operator lifts the external object up to the critical configuration 2 to release the part;

- phase 6: the operator releases the object staying in the critical configuration 2;

- $\quad$ phase 7: the operator moves back in configuration 1 without the part;

- $\quad$ phase 8: exoskeleton assistance is turned off (configuration 1).

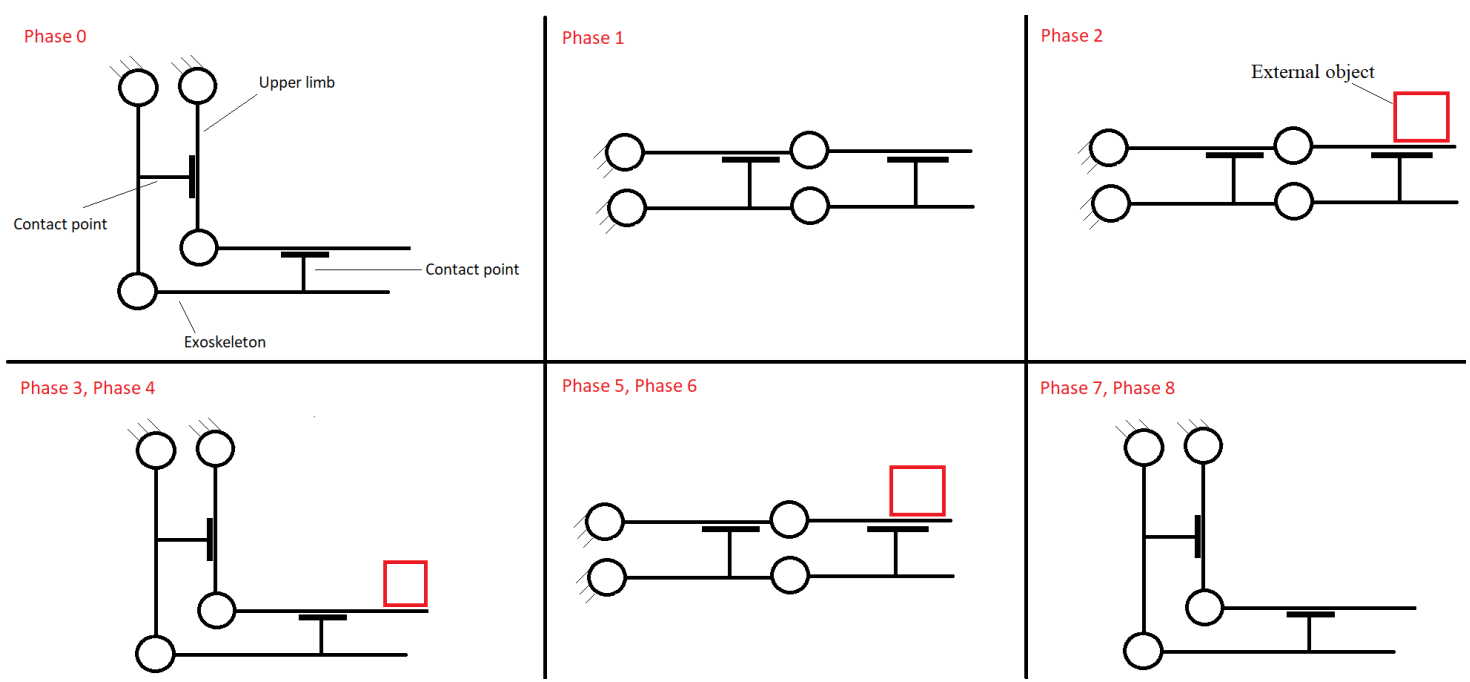

Figure 2. Lifting task phases.

\subsection{Exoskeleton Specifications}

In order to design the exoskeleton to face the proposed task, the following requirements have to be considered:

- kinematics;

- $\quad$ torque requirements; 
- $\quad$ weight and size of the designed device;

- human safety;

- economic affordability.

\subsubsection{Kinematics}

Considering the proposed task and its phases, the kinematics shown in Figure 3 has been proposed for the exoskeleton. In particular, the proposed kinematics is composed by 2 degrees of freedom (DoFs), allowing to perform the complete sequence of task phases while minimizing the DoFs of the device and, therefore, its complexity (i.e., minimizing size, weight and cost of the solution). The Denavit-Hartenberg parameters of the proposed solution are summarized in Table 1.

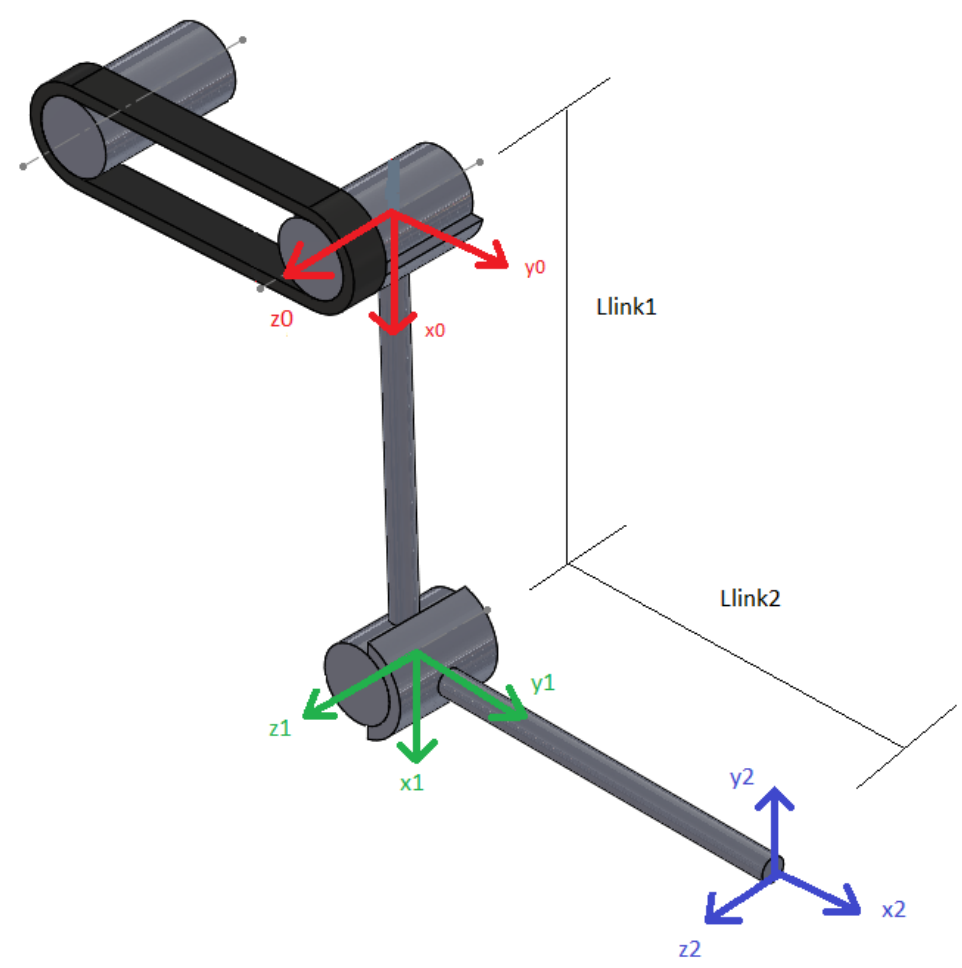

Figure 3. Kinematics of the proposed device.

Table 1. Denavit Hartenberg parameters of the proposed device.

\begin{tabular}{cccc}
\hline $\boldsymbol{\theta}$ & $\boldsymbol{\alpha}$ & $\mathbf{r}$ & $\mathbf{d}$ \\
\hline$\theta_{1}$ & 0 & $L_{\text {linka }}$ & 0 \\
\hline$\theta_{2}$ & 0 & $L_{\text {linkf }}$ & 0 \\
\hline
\end{tabular}

\subsubsection{Torque Requirements}

In order to define the torque requirements associated to the reference task, the most critical configuration assumed by the exoskeleton has to be considered. Therefore, configuration 2 will be used in order to calculate the required motor torques. The applied motor torque has to compensate for the lifted load $P_{\text {load }}$ and the human arm weight (composed by the human arm weight $P_{1}$ and by the human forearm and hand weight $P_{2}$ ), while applying the required assistance to the human. The exoskeleton will, therefore, apply two forces on the upper limbs due to arm support and forearm support, assisting the worker during the target task execution. These forces compensate the external torques applied by the load, therefore, having the human generating no torques. 
Figure 4 shows the exoskeleton-upper limb model, highlighting all the acting forces/torques, where:

- $R_{a}$ : interaction force between the forearm and the exoskeleton;

- $R_{b}$ : interaction force between the arm and the exoskeleton;

- $R_{s}$ : vertical reaction force of the shoulder;

- $a_{1}$ : application point of the $R_{b}$ force;

- $\quad a_{2}$ : application point of the $R_{a}$ force;

- $L_{\text {arm }}$ : human's arm length;

- $L_{f+h}$ : human's forearm and hand;

- $P_{\text {link }}:$ weight of the forearm exoskeleton link applied in the middle of the link;

- $\quad P_{\text {motor }}$ : weight of the elbow motor;

- $P_{\text {link }_{1}}$ : weight of the arm link applied in the middle of the link;

- $\tau_{B}$ : torque of the elbow motor to support the joint;

- $\tau_{\text {mot }}$ : torque of the shoulder motor to support the joint;

- $T_{s}$ : vertical reaction force of the support connecting the exoskeleton link to the back-plate.

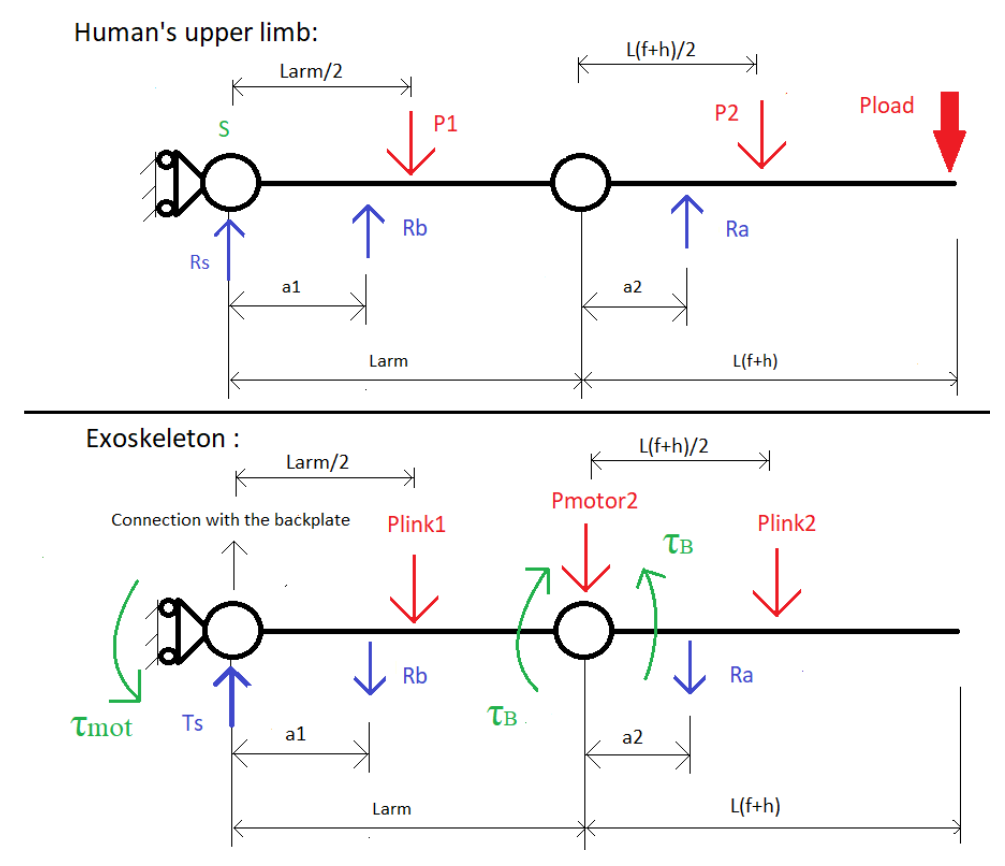

Figure 4. Loads acting on the exoskeleton in the critical configuration 2.

On the basis of Reference [42] (detailing the average height of European adults) and on the basis of Reference [43] (detailing the average weight of European adults), the following parameters have been chosen in order to define the human arm weight and length to be used in the above modeling:

- Height $=H=180 \mathrm{~cm}$.

- Weight $=W=80 \mathrm{~kg}$.

From the references above, it is therefore possible to calculate the modeling parameters from anthropometric tables [44]:

- $\quad L_{\text {arm }}=0.2898 \mathrm{~m}$.

- $\quad L_{\text {forearm }+ \text { hand }}=0.4536 \mathrm{~m}$.

- $w_{\text {arm }}=2.24 \mathrm{~kg}\left(P_{1}=22 \mathrm{~N}\right)$. 
- $w_{\text {forearm }+ \text { hand }}=1.76 \mathrm{~kg}\left(P_{2}=17.27 \mathrm{~N}\right)$.

Assuming that (from a preliminary design) the weight of exoskeleton links is $0.5 \mathrm{~kg}$ (i.e., $\left.P_{\text {link }}=P_{\text {link }_{2}}=4.905 \mathrm{~N}\right)$, the weight of the elbow motor is $1 \mathrm{~kg}\left(P_{\text {motor }_{2}}=9.81 \mathrm{~N}\right), a_{1}=\frac{L_{\text {arm }}}{2}$ and $a_{2}=L_{f+h}$, it is possible to calculate reaction forces and the maximum static torques to be applied by the shoulder and elbow motors considering configuration 2 (Table 2). On the basis of such torque requirements, motors can be selected.

Table 2. Reaction forces and motor torques calculated to compensate for human arm weight and component weight in the critical configuration 2.

\begin{tabular}{cccccc}
\hline$R_{a} \mathbf{N}$ & $R_{b} \mathbf{N}$ & $T_{s} \mathbf{N}$ & $R_{s} \mathbf{N}$ & $\tau_{\text {mot }} \mathbf{N m}$ & $\tau_{B} \mathbf{N m}$ \\
\hline 57.68 & 39.29 & 116.56 & -8.63 & 54.7 & 27.3 \\
\hline
\end{tabular}

\subsubsection{Weight and Size of the Designed Device}

Considering that the upper limbs exoskeleton has to be transported by the human worker, its weight and size have to be reduced as much as possible. Therefore, the selection of the components (e.g., motors) has to consider also such goal. Moreover, in order to limit the size of the designed device (in particular lateral dimension of the device) while ensuring the torque requirements, the shoulder motor has been placed on the back support of the exoskeleton. Mechanical design of the links, etc, also considers both weight and size requirements.

\subsubsection{Human Safety}

In order to improve the transparency and the safety of the device while collaborating with the human, compliance has been embedded into the mechanical structure of the exoskeleton. In particular, a compliant transmission has been designed to connect the shoulder motor to the shoulder link. Such transmission has to be capable to produce an equivalent shoulder stiffness $K_{e q} \in[150,200] \mathrm{Nm} / \mathrm{rad}$. Such equivalent stiffness is a medium-level value capable to give a degree of compliance to the exoskeleton while avoiding too high deformations.

\subsubsection{Economic Affordability}

Components from the market have to be selected both satisfying the previous design requirements and the costs constraints). In fact, one of the main goal of the proposed design is to have hardware costs $<10,000$ Euro. Therefore, a balance between design specifications and hardware costs has to be found.

\section{Exoskeleton Modeling \& Design}

\subsection{Compliant Shoulder Joint Actuation Modeling}

As mentioned in Section 2.2, the proposed exoskeleton design includes a compliant actuation for the shoulder joint. Such actuation system is composed by a compliant belt as a transmission between the motor and the shoulder joint. The concept of the compliant belt actuator is shown in Figure 5. 


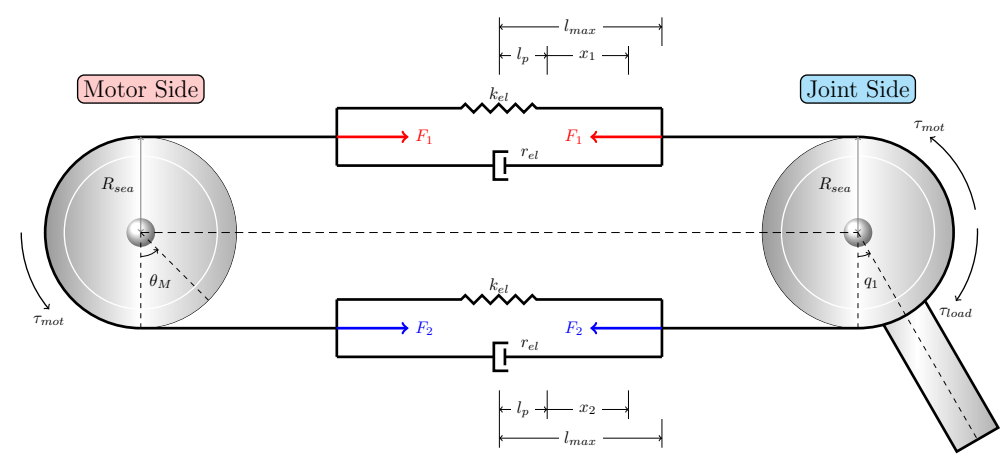

Figure 5. Mechanical model of the compliant belt shoulder joint actuation.

The aforementioned actuation system is modeled as the parallel of two mass-less spring-damper elements (with same stiffness $k_{e l}$ and damping $r_{e l}$ parameters) as in Reference [45]. Being the radius of the pulleys both equal to $R_{\text {sea }}=0.04 \mathrm{~m}$, the deformation of each spring-damper element $x_{i}$ is given by: $x_{1}=-R_{\text {sea }}\left(q_{1}-\theta_{M}\right)$ and $x_{2}=R_{\text {sea }}\left(q_{1}-\theta_{M}\right)=-x_{1}$, where $q_{1}$ is the link-side position and $\theta_{M}$ is the motor-side position as shown in Figure 6. In case of zero motor torque $\tau_{m o t}=0$, namely in the equilibrium position, the deformations are zero.

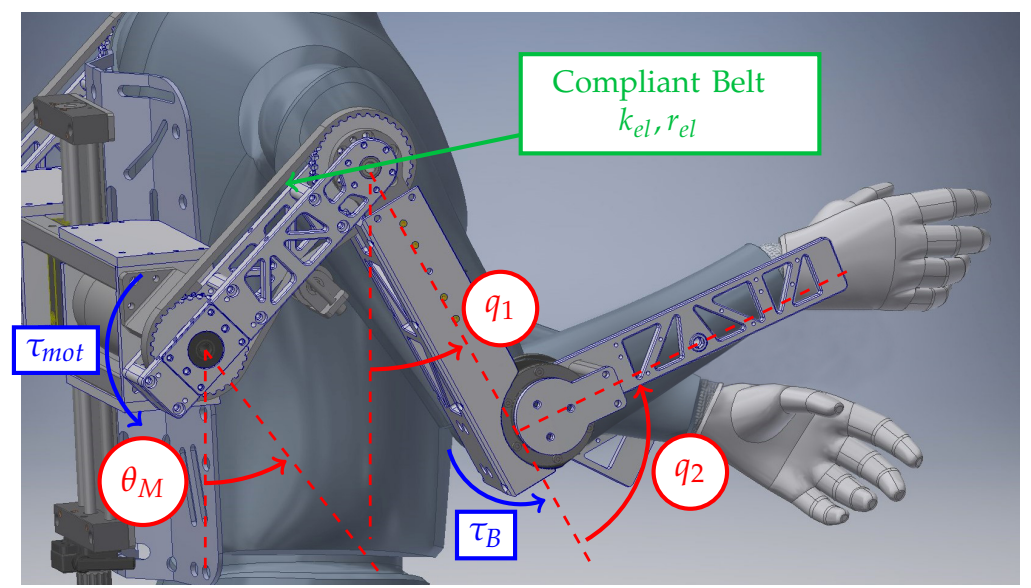

Figure 6. Elastic belt and joint torques are highlighted on the proposed exoskeleton model.

Overall torque transmission is given by the effect of pulling forces $F_{i}$ on the two springs. Being $l_{p_{i}}$ the pre-loaded lengths (equal for both springs), the forces are computed as $F_{i}=k_{e l}\left(l_{p}+x_{i}\right)+r_{e l} \dot{x}_{i}$. Therefore, the relation between motor torques and elastic belt deformations can be derived:

$$
\tau_{m o t}=-2 R_{\text {sea }}^{2} k_{e l}\left(q_{1}-\theta_{M}\right)-2 R_{\text {sea }}^{2} r_{e l}\left(\dot{q}_{1}-\dot{\theta}_{M}\right) .
$$

\subsection{Exoskeleton Dynamic Model}

The overall mechanical system can be represented as a 3-DoFs system that moves in the sagittal plane. The device DoFs are represented by $\theta_{M}$ (the shoulder motor joint position), $q_{1}$ (the shoulder joint position) and $q_{2}$ (the exoskeleton elbow joint position). $\theta_{M}$ and $q_{1}$ are connected by the elastic belt, transmitting the torque by the relation (1). According to the Euler-Lagrangian formulation, the dynamics of the exoskeleton connected to the human arm can be expressed as follows:

$$
\boldsymbol{M}(\boldsymbol{q}) \ddot{\boldsymbol{q}}+\boldsymbol{C}(\boldsymbol{q}, \dot{\boldsymbol{q}})+\boldsymbol{G}(\boldsymbol{q})+\boldsymbol{f}(\dot{\boldsymbol{q}})+\boldsymbol{K}(\boldsymbol{q})+\boldsymbol{D}(\dot{\boldsymbol{q}})=\boldsymbol{\tau}-\boldsymbol{J}_{e}^{T} \boldsymbol{F}_{e},
$$

where:

- $\boldsymbol{q} \in \boldsymbol{R}^{3 x 1}$ is the vector of the DoFs $\left[\theta_{M}, q_{1}, q_{2}\right]^{T}$; 
- $\quad \boldsymbol{M}(\boldsymbol{q}) \in \boldsymbol{R}^{3 \times 3}$ is the system inertia matrix;

- $C(q, \dot{q}) \in R^{3 x 1}$ is the Coriolis and centrifugal vector;

- $G(q) \in R^{3 x 1}$ is the gravitational vector;

- $f(q, \dot{\boldsymbol{q}}) \in R^{3 x 1}$ is the vector of friction forces;

- $\quad K(\boldsymbol{q}) \in \boldsymbol{R}^{3 \times 1}$ is the system elasticity vector;

- $\boldsymbol{D}(\dot{\boldsymbol{q}}) \in \boldsymbol{R}^{3 \times 1}$ is the system damping vector;

- $\tau \in R^{3 x 1}$ is the vector of applied torques at the actuated joints;

- $J_{e}^{T}$ is the transposed extended Jacobian matrix;

- $\quad \boldsymbol{F}_{e}$ is the vector of external forces applied by the human and/or external load.

The formulation in (2) represents three coupled second-order differential equations that relate the joint positions, velocities and accelerations to the joint torques $\tau=\left[\tau_{m o t}, 0, \tau_{B}\right]^{T}$. In particular, $\tau_{m o t}$ is the torque applied by the shoulder motor and $\tau_{B}$ is the torque applied by the elbow motor.

Combining the exoskeleton dynamics of the 2-DoFs manipulator with the compliant belt actuator dynamics in Section 3.1, it is possible to obtain the final overall 3-DoFs configuration. The resulting matrices comprehend terms coming both from the rigid mechanical system and the elastic coupling with the shoulder actuator. Angular velocities and positions of the shoulder joint $q_{1}$ and motor joint $\theta_{M}$ are coupled through the first two elements of the damping vector $\boldsymbol{D}(\dot{\boldsymbol{q}})$ and the stiffness vector $\boldsymbol{K}(\boldsymbol{q})$. The torque transmission to the rigid system is explained in Section 3.1.

Figure 6 represents the CAD model of the exoskeleton and human arm, highlighting the joints (red label), the actuation torques (blue label) and the compliant belt (green label).

\subsection{Compliant Shoulder Joint Design}

In order to select the elastic belt stiffness $k_{e l}$, a preliminary analysis have been performed considering the following stiffness values (peculiar for off-the-shelves elastic belts): $k_{e l}=$ $[25,000,50,000,75,000] \mathrm{N} / \mathrm{m}$. Pre-load has also been varied from zero to the maximum pre-load force $F_{p_{\max }}=k_{e l} \frac{l_{\max }}{2}$ (considering 5 values in such range). By increasing $k_{e l}$, both the maximum transmittable torque and the equivalent shoulder joint stiffness increase (where the equivalent shoulder joint stiffness can be calculated as $\left.K_{e q}=\frac{\partial \tau_{\text {mot }}}{\partial\left(q_{1}-\theta_{M}\right)}=2 k_{e l} R_{\text {sea }}^{2}\right)$.

To select the elastic belt, a shoulder equivalent stiffness $K_{e q} \in[150,200] \mathrm{Nm} / \mathrm{rad}$ has been imposed. Such equivalent stiffness is a medium-level value capable to give a degree of compliance to the exoskeleton while avoiding too high deformations. To satisfy the above requirement, $k_{e l}$ has been selected equal to $50,000 \mathrm{~N} / \mathrm{m}$. Figure 7 a shows the stiffness characteristic curve of the selected ELATECH ${ }^{\circledR}$ SIT Spa belt and Figure $7 \mathrm{~b}$ its equivalent shoulder joint stiffness. The stiffness characteristic variation is due to the pre-load applied to the belt. 


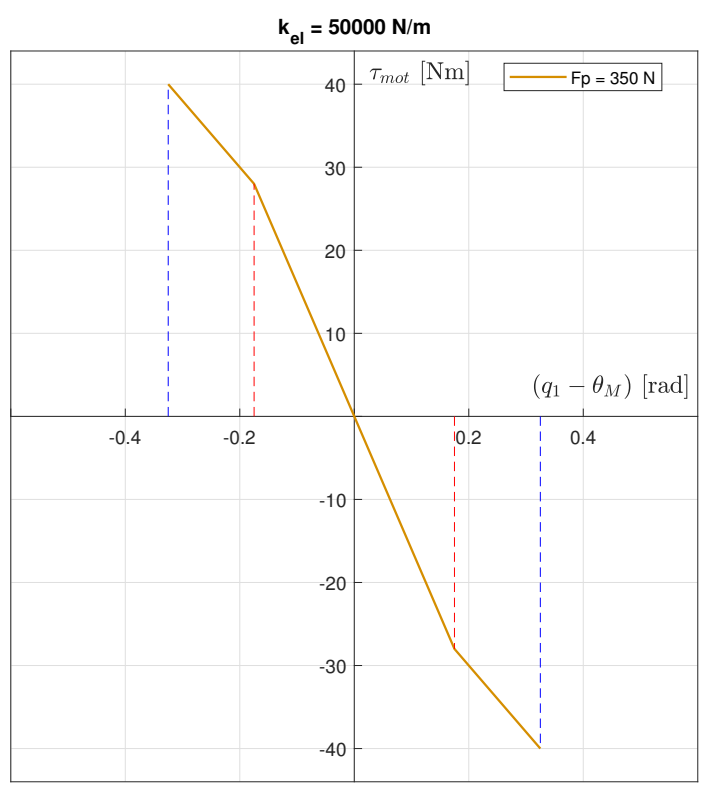

(a)

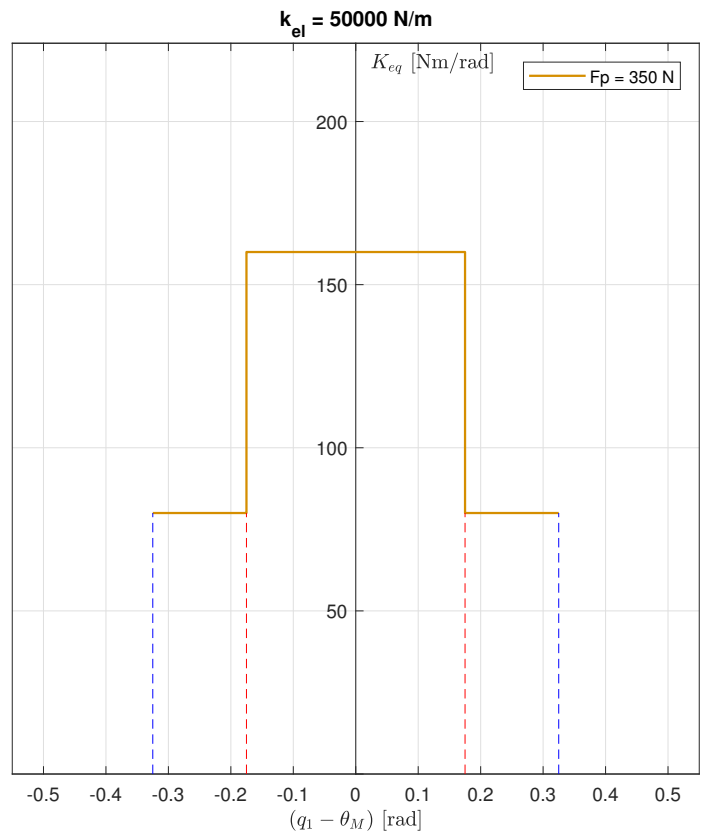

(b)

Figure 7. (a) Stiffness characteristic of the chosen belt. The stiffness characteristic variation is due to the pre-load applied to the belt. (b) Equivalent stiffness at the link-side shoulder joint.

\subsection{Design Solution}

The 2 DoFs kinematics has been selected, with the Denavit-Hartenberg parameters in Table 1.

In the presented section, the selected hardware components are detailed, satisfying the above define design specifications.

\subsubsection{Brushless Motor Maxon EC60 FLAT/MILE/PM72}

The Maxon motor EC60 FLAT/MILE/PM72 (24 V) has been selected as the shoulder motor, including:

- $\quad$ speed reducer IMS PM 72 C Ø72 mm, 4 stage, ratio 305:1.

- EC60 flat, brushless, with Hall sensors.

- encoder Maxon MILE, 1-1024 pulses, 3 differential channels.

- nominal torque $56.30 \mathrm{Nm}$.

The proposed motor satisfy the required static torque within its nominal torque and allows to apply higher pick torques for control purposes. The weight of the motor is $1.5 \mathrm{~kg}$.

\subsubsection{Transmission}

The elastic belt ELATECH ${ }^{\circledR}$ SIT Spa has been selected. The belt has a bielicoidal teeth with a progressive and continuous meshing in order to reduce vibrations and noises. 1 to 1 ratio (EGLE) pulleys have been selected.

\subsubsection{Elbow Actuator}

robolink ${ }^{\circledR} D$ High End robotic joint, size 20, symmetrical, provided by Igus has been selected as the elbow actuator. This motor is composed by a stepper motor (NEMA17/23/23XL) which drives a revolute joint (code: RL-D-20-101-38-01033). The weight of the motor is $0.9 \mathrm{~kg}$. 


\subsubsection{Exoskeleton CAD}

Figure 8 shows the proposed exoskeleton design. The thickness of the arm link is $70 \mathrm{~mm}$ and the elbow motor is placed inside the link. The shoulder motor is fixed to the back-plate through an aluminium support. The position of the support can be adjusted to increase the device wearability. The shoulder motor torque is transmitted to the human's shoulder through the compliant actuation. The link-side shoulder joint mounts an encoder in order to measure $q_{1}$ and, therefore, the angular deformation $q_{1}-\theta_{M}$. On the basis of such measurement and on the basis of the elastic belt stiffness, the external torque applied by the human/external load can be estimated. Such estimate is used in the control loop for the active assistance definition. The shoulder pulley has to be aligned with the shoulder axis parallel to the frontal plane. In such a way, the flexion/extension degree of freedom is implemented. The elbow motor must be also aligned with the operator's elbow joint to guarantee the elbow rotation. The links length can be regulated in order to adapt the exoskeleton to the user. A range of $\pm 3 \mathrm{~cm}$ has been considered for the adaptability of the links length.
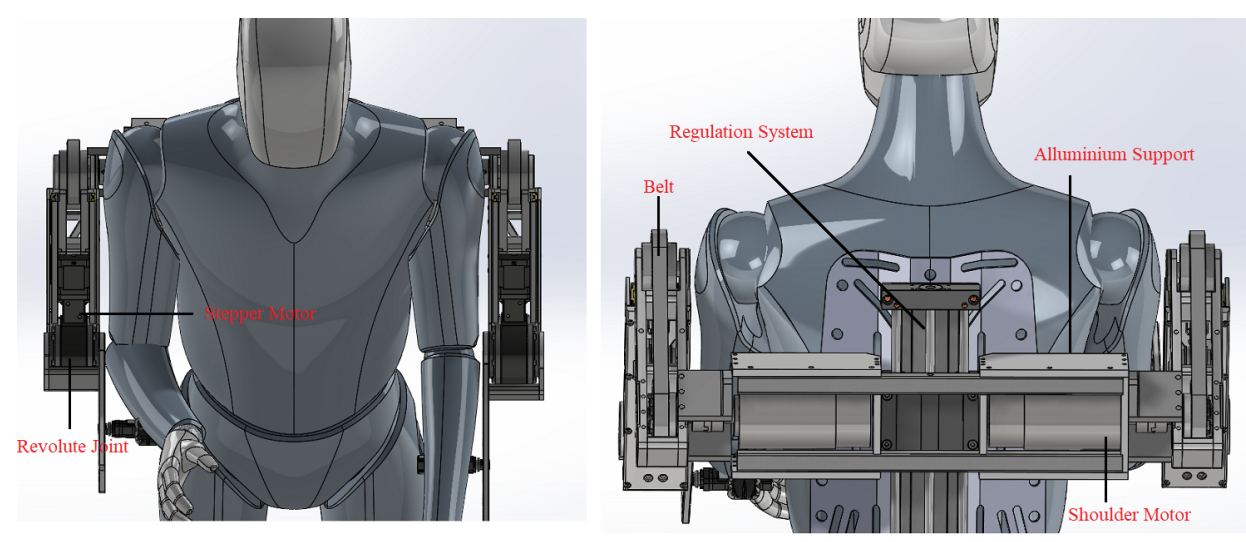

Figure 8. Exoskeleton prototype CAD model.

The total estimated weight of the designed exoskeleton is about $12 \mathrm{~kg}$, not including batteries. The shoulder motors are the heaviest component of the device. On one hand, the weight of the device can be reduced selecting a higher-performance motor for the shoulder joint. However, this will result in higher costs. On the other hand, the exoskeleton payload can be reduced in order to require less performance from motors. Considering the target task detailed in Section 2.1 and considering the budget constraints, the proposed motor is the optimal solution that authors found available on the market in the design phase. It has to be remarked that the payload ratio of the proposed device is $>0.8$.

Remark 1. The above mentioned motors have been selected with a nominal torque satisfying the specifications described in Section 2.2.2. The peak torque is even higher in order to compensate for modeling errors or human subject higher arm weight.

Remark 2. On the basis of the selected motors, the friction model in (2) implements a two parameters friction model for the shoulder and elbow joints $f_{q_{i}}=\alpha_{1, i} \dot{q}_{i}+\alpha_{2, i} \operatorname{sign}\left(\dot{q}_{i}\right)$ (where $i$ indicates the exoskeleton joint) with parameters $\alpha_{1, i}$ and $\alpha_{2, i}$ as in Reference [46] (similar motor as the one mounted on the UR10 manipulator). Such values have been used in simulation. Real friction values have to be identified on the real exoskeleton.

\section{Industrial Exoskeleton Control}

\subsection{Problem Formulation}

The goal of the proposed controller is to assist the human in the lifting and transportation of heavy parts. To achieve such goal, the proposed control logic merges together the perks of the optimal control (to obtain stability and robustness) with the adaptability proposed by the fuzzy logic. A hierarchic 
controller has been designed, composed by an inner optimal control loop (to track a reference task trajectory) and by an outer fuzzy logic control loop (responsible for updating the commanded trajectory according to the detected intentions of motion of the human). The gain scheduling control allows to have online modification of the control gains of the inner optimal controller according to the commanded trajectory. The interaction torque $\tau_{\text {int }}$ between the human and the exoskeleton is calculated exploiting the encoders measurements at both the sides of the elastic belt actuation. $\tau_{\text {int }}$ is exploited by the fuzzy controller in order to identify the intention of motion of the human.

\subsection{Optimal Control Design}

The inner optimal control guarantees the tracking and stabilization of the system around the task reference trajectory. Let the state-space dynamic equations of a linear (or linearized) time-varying system be:

$$
\begin{aligned}
& \dot{\boldsymbol{x}}(t)=A(t) \boldsymbol{x}(t)+\boldsymbol{B}(t) \boldsymbol{u}(t), \\
& \boldsymbol{y}(t)=C(t) \boldsymbol{x}(t),
\end{aligned}
$$

where $x \in \mathbb{R}^{n}$ is the state vector, $A$ and $B$ are the state and input matrix of the linearized system respectively, $\boldsymbol{u} \in \mathbb{R}^{m}$ is the control action vector and $\boldsymbol{y} \in \mathbb{R}^{p}$ is the output vector. According to the optimal control theory on Linear Quadratic Regulator ([47]), it is possible to define a quadratic cost function $J$ as:

$$
J=\frac{1}{2} \Delta \boldsymbol{x}^{T}\left(t_{f}\right) \boldsymbol{P} \Delta \boldsymbol{x}\left(t_{f}\right)+\frac{1}{2} \int_{t_{0}}^{t_{f}}\left(\Delta \boldsymbol{x}^{T}(t) \boldsymbol{Q} \Delta \boldsymbol{x}(t)+\Delta \boldsymbol{u}^{T}(t) \boldsymbol{R} \Delta \boldsymbol{u}(t)\right) d t,
$$

where $\Delta x=q_{r e f}-\boldsymbol{q}, \boldsymbol{P}$ and $\boldsymbol{Q}$ are symmetric and positive semi-definite weight matrices and $\boldsymbol{R}$ is a symmetric and positive definite weight matrix. An optimal LQR controller can be designed in order to minimize this cost function, being the weight matrices previously defined, as shown in Figure 9.

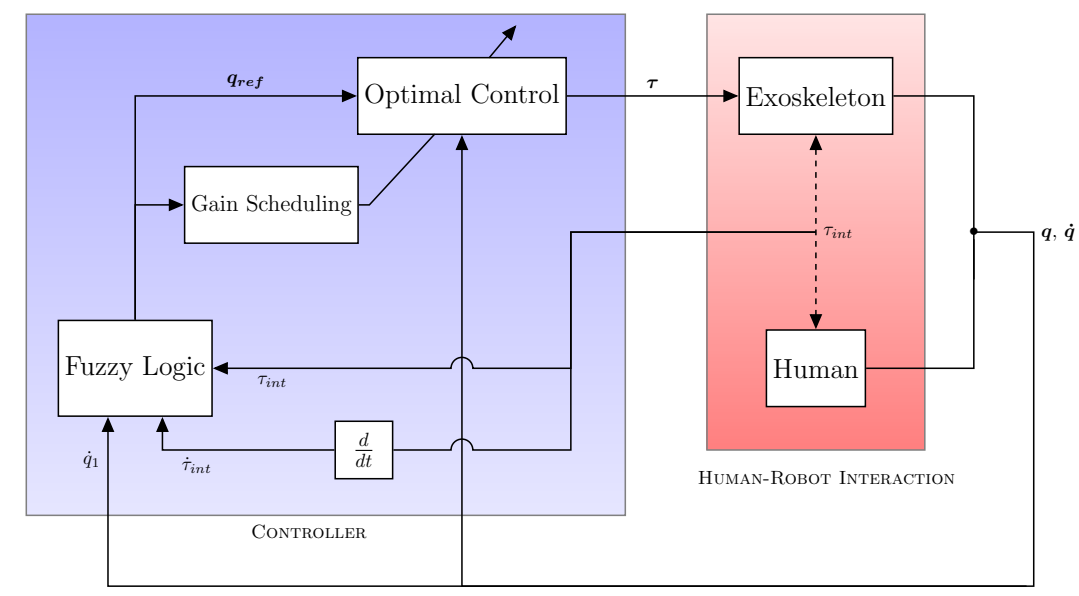

Figure 9. Overall control scheme showing the inner optimal controller, the outer fuzzy logic controller and the gain scheduler, highlighting the feedbacks to the control loops.

\subsection{Gain Scheduling Control Design}

A gain scheduling $L Q R$ approach has been used in order to have a performing and stabilizing controller for all the possible configurations of the system. This offline controller, in fact, modifies the control gain matrix on the basis of the desired pre-defined trajectory. 
Basically, the nonlinear system outlined in Section 3 is quasi-linearized into several linear time-invariant (LTI) subsystems with respect to different operating points. Let the nonlinear state equation of the system be represented as:

$$
\dot{\boldsymbol{x}}(t)=\boldsymbol{\eta}(\boldsymbol{x}(t), \boldsymbol{u}(t), t)=\left[\ddot{\theta}_{M}, \dot{\theta}_{M}, \ddot{q}_{1}, \dot{q}_{1}, \ddot{q}_{2}, \dot{q}_{2}\right]^{T} .
$$

The Taylor series expansion using the appropriate operating points $\left(\boldsymbol{x}^{*}, \boldsymbol{u}^{*}\right)$ can be obtained as $\dot{x}=A \boldsymbol{x}+\boldsymbol{B} \boldsymbol{u}+\boldsymbol{E}_{r r}\left(\boldsymbol{x}^{*}, \boldsymbol{u}^{*}, t\right)$, where:

$$
A=\left.\frac{\partial \eta(x, u)}{\partial x}\right|_{\substack{x=x^{*} \\ u=u^{*}}} \quad \text { and } \quad B=\left.\frac{\partial \eta(x, u)}{\partial u}\right|_{\substack{x=x^{*} \\ u=u^{*}}} .
$$

As demonstrated by Reference [48], the system approximation error term $\boldsymbol{E}_{r r}\left(\boldsymbol{x}^{*}, \boldsymbol{u}^{*}, t\right)$ can be neglected, having the nonlinear system simply linearized as $\dot{\boldsymbol{x}}=\boldsymbol{A} \boldsymbol{x}+\boldsymbol{B} \boldsymbol{u}$.

Due to the fact that the linearization around the operating points holds, the plant and the weight matrices are assumed to be time-invariant and, therefore, $t_{f}$ in the time interval can be assumed to be equal to infinite.

If $\boldsymbol{P}(t)$ does converge, $\dot{\boldsymbol{P}}=\mathbf{0}$ can be assumed for $t \ll t_{f}$ and the resulting equation for the LQR problem is the so-called algebraic Riccati equation (ARE):

$$
\mathbf{0}=-\boldsymbol{Q}-\boldsymbol{P} \boldsymbol{A}-\boldsymbol{A}^{T} \boldsymbol{P}+\boldsymbol{P B} \boldsymbol{R}^{-1} \boldsymbol{B}^{T} \boldsymbol{P} .
$$

Therefore, if $\boldsymbol{P}_{\infty}$ exists, the corresponding steady-state feedback gain matrix is given by $\boldsymbol{K}_{\infty}=\boldsymbol{R}^{-1} \boldsymbol{B}^{T} \boldsymbol{P}_{\infty}$. The resulting optimal control law is then obtained as: $\Delta \boldsymbol{u}^{*}(t)=-\boldsymbol{K} \Delta \boldsymbol{x}(t)$.

The adopted strategy aims to apply the quasi-linearization approach to the several system configurations related to the typical arm lifting trajectory as detailed in Section 2.1. The feedback control gains are computed for each configuration of the exoskeleton. Such control gains are stored and, based on the reference set-point $q_{1}^{r e f}$, online applied by the gain scheduler. The proposed strategy is schematized in Figure 10.

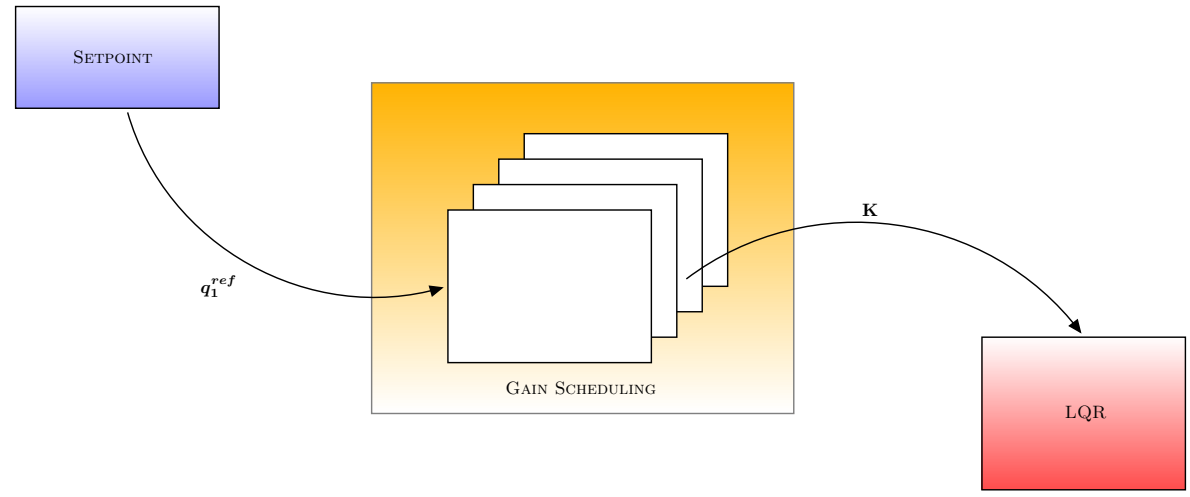

Figure 10. Gain scheduler online updating the control matrix to the inner optimal controller.

\subsection{Empowering Fuzzy Controller Design}

The main motivation to adopt a fuzzy logic for the outer human's intentions-based control is to deal with complex, ill-defined, uncertain and dynamic processes, which are intrinsically difficult to being modelled mathematically. In order to account for human's behaviour inside the control architecture while establishing a human-oriented input-output relations, a fuzzy table is built. The empowering fuzzy controller, therefore, is the highest level controller and it is responsible for the online modification of the reference trajectory, based on the human's intentions of motion. 
The inputs of the developed fuzzy controller are the interaction torque $\tau_{\text {int }}$, the interaction torque derivative $\dot{\tau}_{\text {int }}$ and shoulder joint angular velocity $\dot{q}_{1}$. The output is the assistance level $A_{L}$ that is yielded to the controller in order to modify the reference set-point of the optimal control.

The strategy behind the choice of the inputs membership functions is to obtain an adequate map of the general motion intentions from the human operator, classifying whether the applied torque is intentional or not, thus recognizing if the shoulder joint is willing to move from the current configuration or not. At the same time, an appropriate definition of safety between human and exoskeleton is achieved. More in details, the speed of the shoulder and the interaction torque are monitored to avoid the excess of the specified threshold. Moreover, the embedded strategy allows to recognize whether the human's intention is willing to lift (lifting assistance) or lower (lowering assistance) the arm, accordingly defining the deformation of the optimal control trajectory.

\subsubsection{Membership Functions}

The states of the membership function characterizing the shoulder joint velocity are: stop, slow, move and fast, whose aim is to decompose the velocity range into different states, to know whether the shoulder angular variation is too fast, too slow, in the desired motion range or to understand if the human wants to stop the motion. The states of the membership function characterizing the interaction torque are: $N, S$ and NS, which stand respectively for no torque (below a specified threshold the control is not activated), safe and not safe. Finally, the states of the membership function characterizing the interaction torque derivative are: variation $(V)$ or no variation $(N V)$, meaning if the operator wants to move the shoulder or change the motion by increasing or reducing the applied torque or not.

The output membership functions for the assistance level $A_{L}$ range over four different fuzzy sets: none, low, medium and high.

\subsubsection{Rule Base}

The rule base for the current control strategy can be summed up with the following nine rules for the lifting assistance and eight rules for the lowering assistance (rule \#8 is omitted for the lowering phase). The rule base adjusts the level of assistance depending on how much the operator is willing to move from the current arm position, respectively yielding null to high assistance to the shoulder motion:

$$
\begin{cases}\# 1 & \text { IF } \dot{q}_{1} \text { is stop AND } \tau_{\text {int }} \text { is } S \text { AND } \dot{\tau}_{\text {int }} \text { is } N V \text { THEN } A_{L} \text { is none } \\ \# 2 & \text { IF } \dot{q}_{1} \text { is stop AND } \tau_{\text {int }} \text { is } S \text { AND } \dot{\tau}_{\text {int }} \text { is } V \text { THEN } A_{L} \text { is low } \\ \# 3 & \text { IF } \dot{q}_{1} \text { is slow AND } \tau_{\text {int }} \text { is } S \text { AND } \dot{\tau}_{\text {int }} \text { is NV THEN } A_{L} \text { is low } \\ \# 4 & \text { IF } \dot{q}_{1} \text { is slow AND } \tau_{\text {int }} \text { is } S \text { AND } \dot{\tau}_{\text {int }} \text { is } V \text { THEN } A_{L} \text { is medium } \\ \# 5 & \text { IF } \dot{q}_{1} \text { is move AND } \tau_{\text {int }} \text { is } S \text { AND } \dot{\tau}_{\text {int }} \text { is NV THEN } A_{L} \text { is medium } \\ \# 6 & \text { IF } \dot{q}_{1} \text { is move AND } \tau_{\text {int }} \text { is } S \text { AND } \dot{\tau}_{\text {int }} \text { is } V \text { THEN } A_{L} \text { is high } \\ \# 7 & \text { IF } \dot{q}_{1} \text { is fast THEN } A_{L} \text { is none } \\ \# 8 & \text { IF } \tau_{\text {int }} \text { is NS THEN } A_{L} \text { is none } \\ \# 9 & \text { IF } \tau_{\text {int }} \text { is N THEN } A_{L} \text { is none. }\end{cases}
$$

Figure 11 shows the process of fuzzy rule base for the lifting assistance developed in MATLAB ${ }^{\circledR}$. 


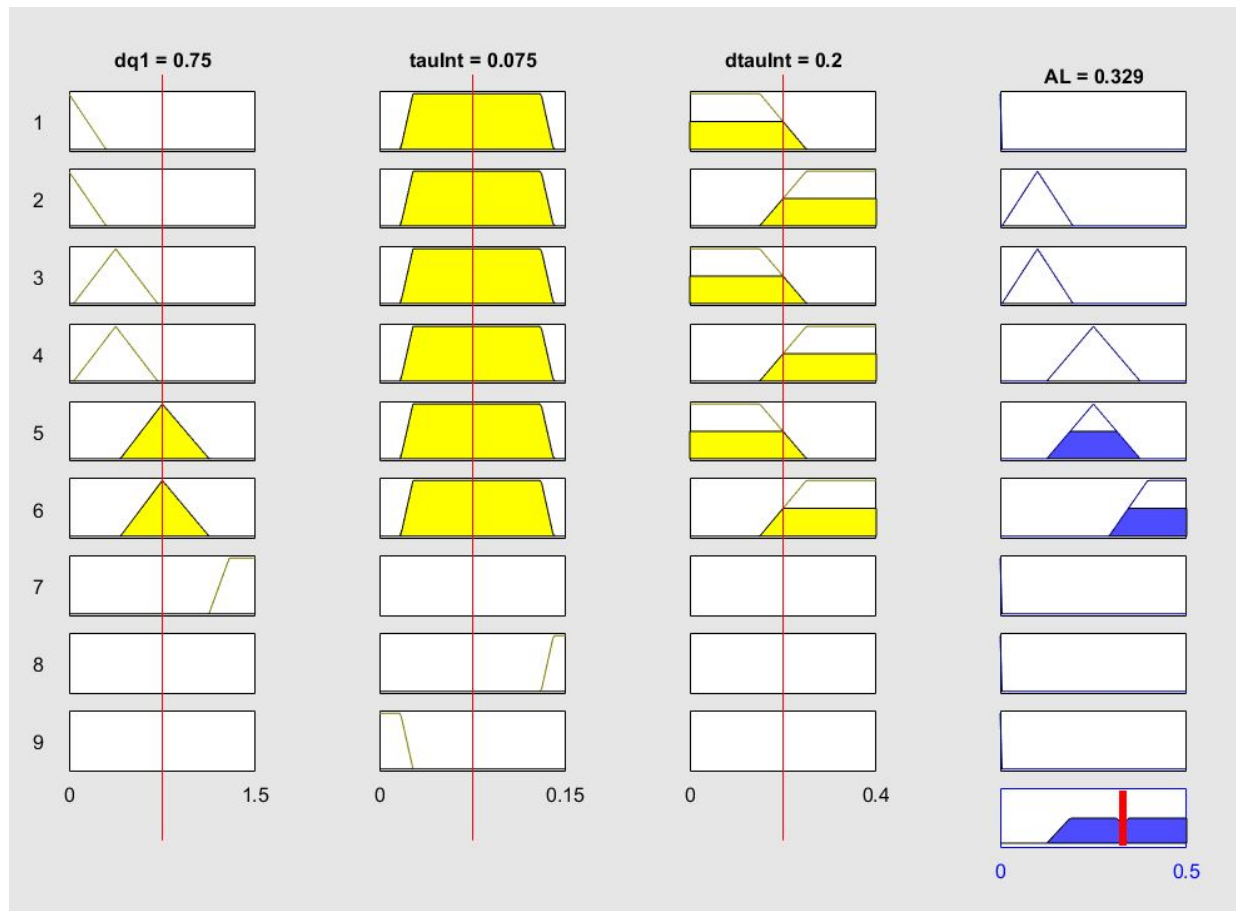

Figure 11. The nine fuzzy rules for the lifting assistance represented in MATLAB ${ }^{\circledR}$.

The online trajectory generation to be provided to the inner optimal controller and gain scheduler is, therefore, deformed by the required assistance by the human:

$$
q_{1}^{r e f}=q_{1}-A_{L} \operatorname{sign}\left(\tau_{i n t}\right) .
$$

In particular, the shoulder reference angular position is computed from the stored value of $q_{1}$ that is updated with a frequency of $50 \mathrm{~Hz}$, only if the value of assistance level is different from zero. The value $A_{L} \operatorname{sign}\left(\tau_{\text {int }}\right)$ allows to establish whether the reference set-point needs to be decreased or increased with respect to the previous value, by considering the sign of the interaction torque.

Remark 3. It has to be underlined that the only use of the force measurements cannot allow the fuzzy logic to identify an intention of motion of the human (the weight of the arm/payload affects this estimation). Including the velocity and the force derivative in the fuzzy controller can instead allow to understand if the human is intended to move the exoskeleton. In this case, in fact, it is possible to monitor the full interaction state between the human and the robot.

Remark 4. The proposed controller extends the work in Reference [49] including improved fuzzy membership functions and rules, together with the proposed lower level gain scheduling optimal controller.

\section{Simulation Validation}

The effectiveness of the proposed control architecture has been validated in simulation. All the analyses have been carried out considering the nominal parameters of the system (Section 2.2), affected by uncertainties, in order to take into account modeling errors (e.g., unknown upper limb anthropometric characteristics, involuntary tremor, etc.).

The simulations are performed using MATLAB ${ }^{\circledR} /$ Simulink.

\subsection{Empowering Human in Lifting Task}

The proposed controller has been tested in simulation assisting the operator in a lifting task of a $10 \mathrm{~kg}$ part (i.e., $5 \mathrm{~kg}$ for each exoskeleton arm as defined in Section 2.1). In the proposed simulation the 
payload is grasped at time equal to $1 \mathrm{~s}$. At time equal to $4.5 \mathrm{~s}$ a torque is applied by the human shoulder to interact with the exoskeleton. Three interaction torque levels are simulated in Figure 12: $8 \mathrm{Nm}$, $12 \mathrm{Nm}$ and $16 \mathrm{Nm}$. Such three torque levels have been implemented in simulation in order to show the different activation of the fuzzy rules. The simulation considers an initial joint velocity equal to zero (i.e., the corresponding state of the velocity membership function is stop). All the applied torques make the derivative of the interaction torque resulting in the variation state $V$. Considering the applied torques, the first interaction torque level is not able to activate the fuzzy controller to assist the human (i.e., the corresponding state of the interaction torque membership function is no torque $N$ ). The second and third interaction torque levels, instead, activate the assistance (i.e., the the corresponding state of the interaction torque membership function is safe S), deforming the set-point to the optimal controller until the torque decreases to zero. The proposed controller is, therefore, capable to distinguish from required assistance, empowering the human operator.

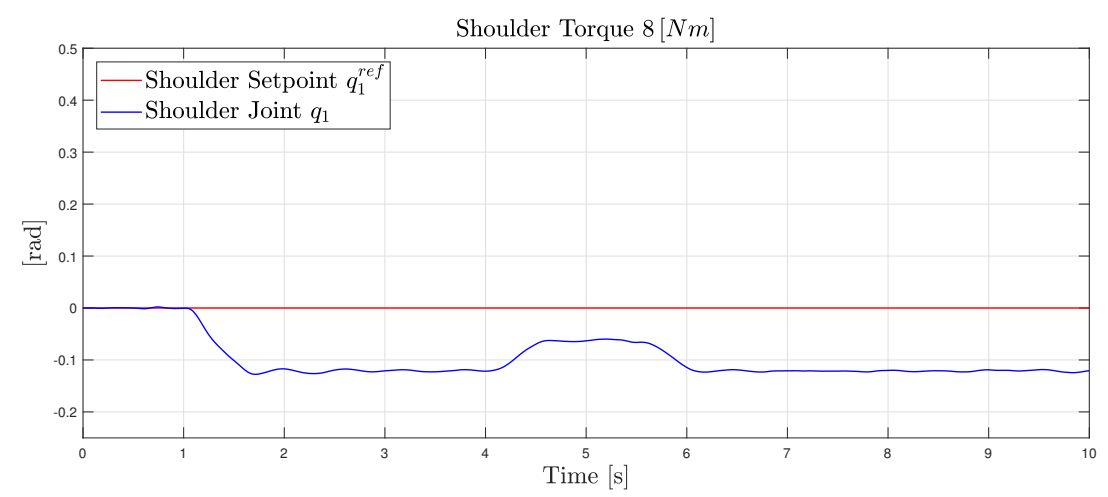

(a)

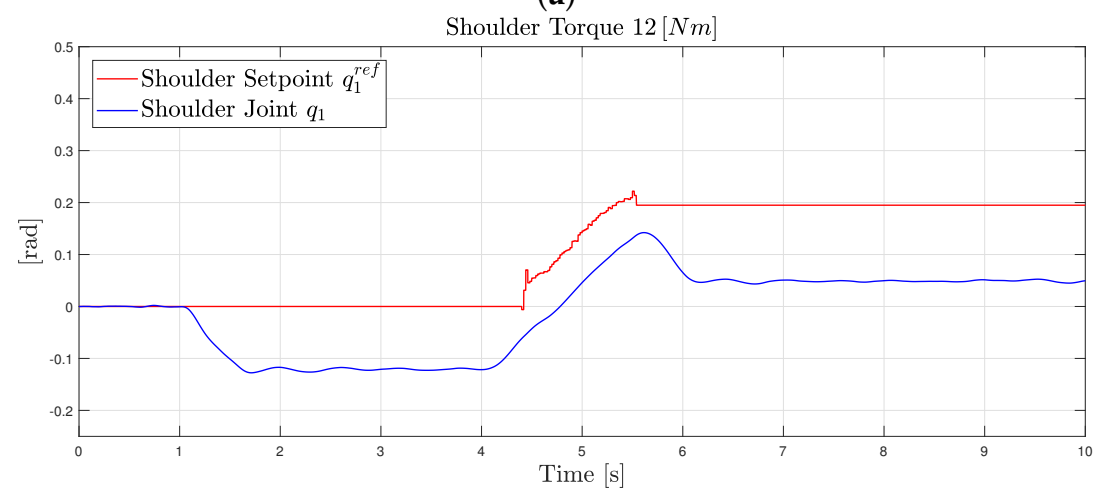

(b)

Figure 12. Cont. 


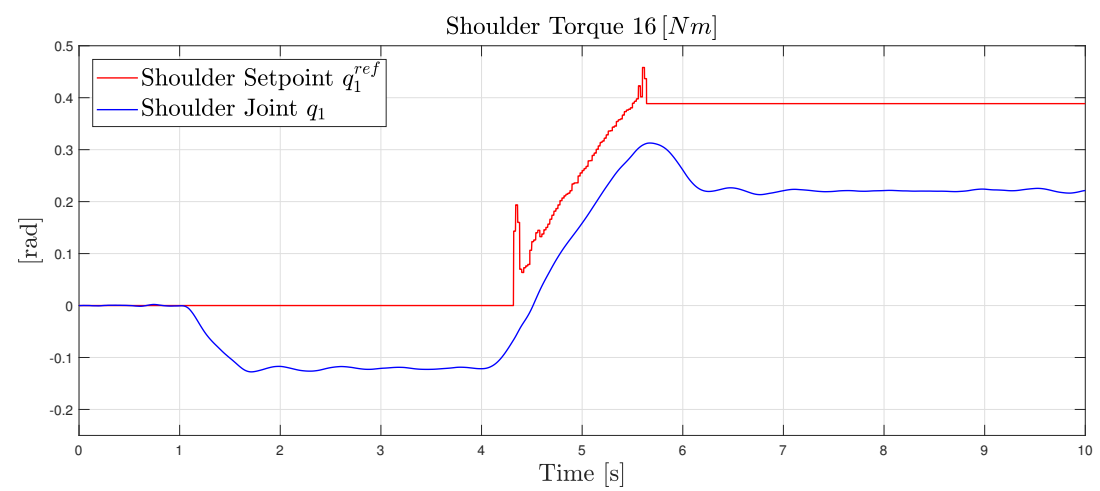

(c)

Figure 12. (a) The interaction torque level $8 \mathrm{Nm}$ is applied while manipulating the $10 \mathrm{~kg}$ payload added (i.e., $5 \mathrm{~kg}$ for each exoskeleton arm as defined in Section 2.1). The applied interaction torque is not able to activate the fuzzy controller to deform the set-point to the optimal control to assist the operator. (b) and (c) are related respectively to the $12 \mathrm{Nm}$ and $16 \mathrm{Nm}$ interaction torque levels. The fuzzy controller is activated, empowering the operator. In (c) the assistance is higher then in (b) due to the higher interaction torque level.

\section{2. (Partially) Unknown Part Manipulation Task}

Concerning the real industrial scenario, where the operator lifts and carries an external weight (like a car's bumper, Section 2.1), the proposed exoskeleton control logic has to guarantee an adequate support to the arm even manipulating (partially) unknown weight parts. The here presented simulation evaluates the performance of the model-based controller for an unknown load-handling task scenario.

The simulation in Figure 13 shows the shoulder joint position time history when an unknown external weight of $4 \mathrm{~kg}$ is applied on the exoskeleton (at time $t=1 \mathrm{~s}$ ). The proposed controller has been compared with a PID controller that compute the control action $\tau_{P I D}$ as follows:

$$
\tau_{P I D}(t)=K_{p}\left(q_{1}^{r e f}(t)-q_{1}(t)\right)+K_{d}\left(\dot{q}_{1}^{r e f}(t)-\dot{q}_{1}(t)\right)+K_{i} \int\left(q_{1}^{r e f}(t)-q_{1}(t)\right) d t
$$

where $K_{p}=50 \mathrm{Nm} / \mathrm{rad}$ is the proportional gain, $K_{d}=15 \mathrm{Nms} / \mathrm{rad}$ is the derivative gain and $K_{i}=4 \mathrm{Nm} / \mathrm{rad} / \mathrm{s}$ is the integral gain. The gains have been experimentally determined to achieve the maximum simulation performance while maintaining the system stability.

The fuzzy logic controller identifies that such load application is not resulting from a human intention of motion, therefore, not updating the reference trajectory to the inner optimal controller and gain scheduler. Comparing the results with the PID controller, the proposed controller is capable to suppress the vibrations while resulting in less deformation of the joint position.

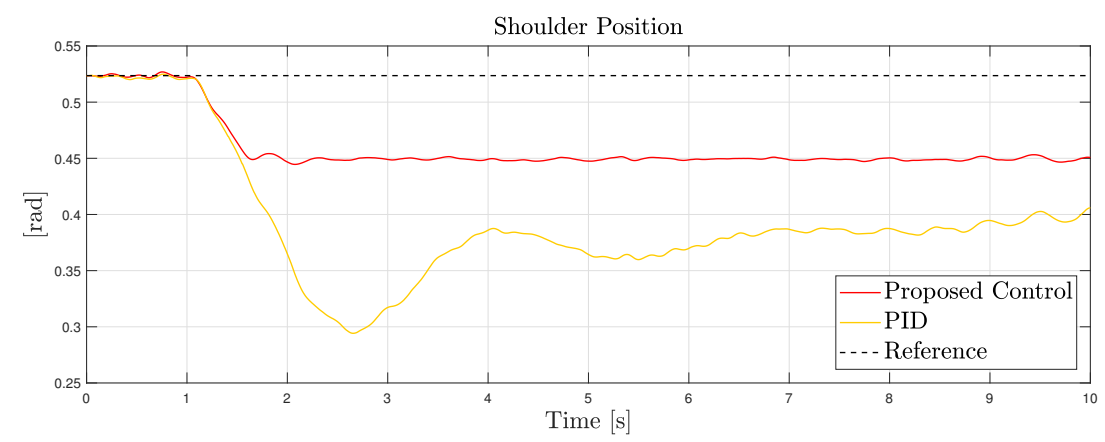

Figure 13. Comparison between the proposed controller and PID controller compensating a $4 \mathrm{~kg}$ payload unknown weight. The proposed controller is capable to suppress vibrations, resulting in less deformation. 


\section{Discussion}

The proposed methodology have considered both mechanical design and control design of a low-cost industrial upper limbs exoskeleton. On the basis of the provided analysis, it has been possible to define the mechanical design specifications, taking into account the reference task. Off-the-shelves components (such as motors, elastic belt, etc.) have been identified to satisfy the design requirements. In particular, the belt has been selected to satisfy the compliance actuation requirements, in order to ensure safety at the hardware level. It has to be underlined that a trade-off between design requirements and available solutions is required. In particular, the economic requirement and the torque requirements have been prioritized, with respect to weight and size requirements. The resulting solution, in fact, has a weight of $12 \mathrm{~kg}$ without batteries. The device weight can be reduced of about $3 \mathrm{~kg}$ by changing the shoulder motors (e.g., purchasing the ANYdrive actuators [50]). However, different motors will increase considerably the hardware costs (costs can vary from 1500 Euro to 5000 Euro per motor). The empowering controller has been designed with emphasis on safety. Safety rules embedded in the outer empowering fuzzy logic have been design on the basis of the interaction state. The designed safety rules allow to achieve a trade-off between empowering performance and safety requirements, avoiding to high dynamics motions, while guaranteeing a safe human-robot interaction. The inner optimal gain scheduling controller allows for the tracking of the reference provided by the outer control loop.

\section{Conclusions}

The here presented paper describes the mechanical and control design solutions for (i) a low-cost hardware industrial exoskeleton (ii) with high payload ratio to be adopted in lifting and transportation of heavy parts. Mechanical design specifications have been derived from the task, allowing to design (iii) an intrinsic compliant 2 DoFs exoskeleton exploiting e SEA actuation at the shoulder joint to intrinsically increase human-robot interaction safety. The proposed control architecture has been described, defining (iv) a safety-based control framework. The inner gain scheduling optimal controller allows for task trajectory tracking. The outer safety-based fuzzy logic controller allows for human empowering. Simulation results show promising performance in the assistance of human operators (damping vibrations and empowering workers) and in the manipulation of unknown payloads.

The prototype of the proposed solution is under realization and it will be experimentally tested in the proposed task to evaluate the proposed approach. In particular, 20 subjects will be considered in the experimental tests. Both cognitive evaluation (based on questionnaires) and quantitative evaluation (based on EMG measurements) will be performed as in Reference [49]. In addition, authors will apply to the second call of the EUROBENCH project to test the proposed exoskeleton in the EUROBENCH exoskeletons benchmark facility, where the leading author Roveda is also leading the STEPbySTEP project [51].

\section{Current and Future Work}

Considering the mechanical design of the device, current work is focusing on a 3 DoFs shoulder joint concept, implementing 2 additional passive DoFs. In this new concept, the shoulder motor is considered aligned with the shoulder joint. The resulting exoskeleton implements, therefore 4, DoFs (Figure 14). The main advantage of the proposed new design is related to the increased mobility of the shoulder. However, such joint requires a different design of the compliant actuation. Therefore, the here mentioned solution is still under evaluation. Additional work is devoted to design a passive ergonomic back support for the exoskeleton to increase the ergonomics of the device. Considering the control design of the device, machine learning techniques are investigated to optimize the outer controller parameters. 


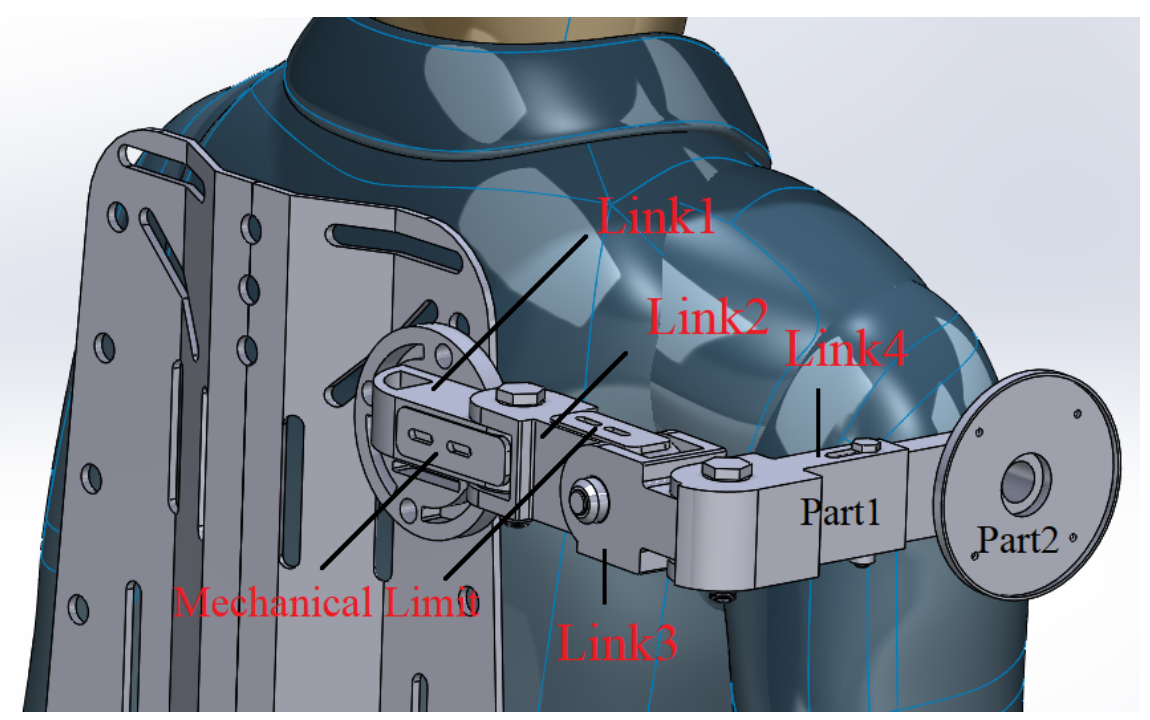

Figure 14. Three DoFs shoulder joint: new concept to increase exoskeleton mobility and task DoFs.

Author Contributions: Conceptualization, L.R., J.L., G.F., D.F., M.M. and A.M.; methodology, L.R.; software, A.M., J.L. and L.R.; validation, A.M., J.L. and L.R.; formal analysis, A.M., J.L. and L.R.; investigation, J.L. and L.R.; resources, L.R.; data curation, L.R.; writing-original draft preparation, J.L., A.M. and L.R.; writing-review and editing, L.R.; visualization, L.R.; supervision, M.M., F.B. and G.L.; project administration, L.R.; funding acquisition, L.R.

Funding: The work has been developed within the EFFORTLESS project, funded from CNR-STIIMA. This project has received funding from the European Union's Horizon 2020 research and innovation programme, via an Open Call issued and executed under Project EUROBENCH (grant agreement No 779963).

Acknowledgments: Authors would like to thank Tito Dinon (CNR-STIIMA) for his expertise and support in the project.

Conflicts of Interest: The authors declare no conflict of interest.

\section{References}

1. Pons, J.; Rocon, E.; Ruiz, A.; Moreno, J. Upper-limb robotic rehabilitation exoskeleton: Tremor suppression. In Rehabilitation Robotics; Itech Education and Publishing: Vienna, Austria, 2007; pp. 453-470.

2. Lo, H.S.; Xie, S.Q. Exoskeleton robots for upper-limb rehabilitation: State of the art and future prospects. Med. Eng. Phys. 2012, 34, 261-268. [CrossRef] [PubMed]

3. Jha, P.; Savla, K.; Shah, D. Exoskeleton Arm. In Proceedings of the 2018 International Conference on Smart City and Emerging Technology (ICSCET), Mumbai, India, 5 January 2018; pp. 1-6.

4. Bogue, R. Exoskeletons-A review of industrial applications. Ind. Robot Int. J. 2018, 45, 585-590. [CrossRef]

5. De Looze, M.P.; Bosch, T.; Krause, F.; Stadler, K.S.; O'Sullivan, L.W. Exoskeletons for industrial application and their potential effects on physical work load. Ergonomics 2016, 59, 671-681. [CrossRef] [PubMed]

6. Stadler, K.S.; Scherly, D. Exoskeletons in industry: Designs and their potential. In Proceedings of the AUTSYM 2017-8th International Symposium on AUTOMATICCONTROL, Wismar, Germany, 21-22 September 2017; ZHAW Zürcher Hochschule für Angewandte Wissenschaften: Winterthur, Switzerland, 2017.

7. Bosch, T.; van Eck, J.; Knitel, K.; de Looze, M. The effects of a passive exoskeleton on muscle activity, discomfort and endurance time in forward bending work. Appl. Ergon. 2016, 54, 212-217. [CrossRef] [PubMed]

8. Spada, S.; Ghibaudo, L.; Gilotta, S.; Gastaldi, L.; Cavatorta, M.P. Investigation into the applicability of a passive upper-limb exoskeleton in automotive industry. Procedia Manuf. 2017, 11, 1255-1262. [CrossRef]

9. Noonee. Available online: https:/ / www.noonee.com/ (accessed on 8 July 2019).

10. ESOSCHELETRO MATE. Available online: https://www.comau.com/it/mate (accessed on 8 July 2019).

11. Work Safer with EksoVest. Available online: https:/ / eksobionics.com/eksoworks / (accessed on 8 July 2019).

12. Levitate. Available online: https://www.levitatetech.com/ (accessed on 8 July 2019). 
13. Imagine If the Human Body Was Not Limited by Its Physical Abilities? Available online: https://www. skelex.com/(accessed on 8 July 2019).

14. Gopura, R.; Bandara, D.; Kiguchi, K.; Mann, G.K. Developments in hardware systems of active upper-limb exoskeleton robots: A review. Robot. Auton. Syst. 2016, 75, 203-220. [CrossRef]

15. Sylla, N.; Bonnet, V.; Colledani, F.; Fraisse, P. Ergonomic contribution of ABLE exoskeleton in automotive industry. Int. J. Ind. Ergon. 2014, 44, 475-481. [CrossRef]

16. Stadler, K.S.; Altenburger, R.; Schmidhauser, E.; Scherly, D.; Ortiz, J.; Toxiri, S.; Mateos, L.; Masood, J. Robo-mate an exoskeleton for industrial use-Concept and mechanical design. In Advances in Cooperative Robotics; World Scientific: Singapore, 2017; pp. 806-813.

17. Ebrahimi, A. Stuttgart Exo-Jacket: An exoskeleton for industrial upper body applications. In Proceedings of the 2017 10th International Conference on Human System Interactions (HSI), Ulsan, Korea, 17-19 July 2017; pp. 258-263.

18. Li, R.Y.M.; Ng, D.P.L. Wearable Robotics, Industrial Robots and Construction Worker's Safety and Health. In International Conference on Applied Human Factors and Ergonomics; Springer: Cham, Switzerland, 2017; pp. 31-36.

19. Huysamen, K.; de Looze, M.; Bosch, T.; Ortiz, J.; Toxiri, S.; O'Sullivan, L.W. Assessment of an active industrial exoskeleton to aid dynamic lifting and lowering manual handling tasks. Appl. Ergon. 2018, 68, 125-131. [CrossRef]

20. Atoun. Available online: http:/ / atoun.co.jp/products (accessed on 8 July 2019).

21. Innophys. Available online: https://innophys.jp/? (accessed on 8 July 2019).

22. Sankai, Y. HAL: Hybrid assistive limb based on cybernics. In Robotics Research; Springer: Berlin/Heidelberg, Germany, 2010; pp. 25-34.

23. Sarcos. Available online: https://www.sarcos.com/products/guardian-xo/ (accessed on 8 July 2019).

24. Vanderborght, B.; Albu-Schäffer, A.; Bicchi, A.; Burdet, E.; Caldwell, D.G.; Carloni, R.; Catalano, M.; Eiberger, O.; Friedl, W.; Ganesh, G.; et al. Variable impedance actuators: A review. Robot. Auton. Syst. 2013, 61, 1601-1614. [CrossRef]

25. Vitiello, N.; Cempini, M.; Crea, S.; Giovacchini, F.; Cortese, M.; Moisè, M.; Posteraro, F.; Carrozza, M.C. Functional design of a powered elbow orthosis toward its clinical employment. IEEE/ASME Trans. Mechatron. 2016, 21, 1880-1891. [CrossRef]

26. Bianchi, M.; Cempini, M.; Conti, R.; Meli, E.; Ridolfi, A.; Vitiello, N.; Allotta, B. Design of a series elastic transmission for hand exoskeletons. Mechatronics 2018, 51, 8-18. [CrossRef]

27. Yu, H.; Huang, S.; Chen, G.; Pan, Y.; Guo, Z. Human-robot interaction control of rehabilitation robots with series elastic actuators. IEEE Trans. Robot. 2015, 31, 1089-1100. [CrossRef]

28. Li, X.; Pan, Y.; Chen, G.; Yu, H. Adaptive human-robot interaction control for robots driven by series elastic actuators. IEEE Trans. Robot. 2016, 33, 169-182. [CrossRef]

29. Fernández, J.; Sprengel, H.; Mallwitz, M.; Zipper, M.; Yu, B.; Bargsten, V. Designing modular series-elastic actuators for safe human-robot collaboration in industrial settings. In Advances in Cooperative Robotics, Proceedings of the 19th International Conference on Clawar 2016, London, UK, 12-14 September 2016; World Scientific: Singapore, 2016; p. 135.

30. Lenzi, T.; Vitiello, N.; De Rossi, S.M.M.; Roccella, S.; Vecchi, F.; Carrozza, M.C. NEUROExos: A variable impedance powered elbow exoskeleton. In Proceedings of the 2011 IEEE International Conference on Robotics and Automation, Shanghai, China, 9-13 May 2011; pp. 1419-1426.

31. Kim, B.; Deshpande, A.D. An upper-body rehabilitation exoskeleton Harmony with an anatomical shoulder mechanism: Design, modeling, control, and performance evaluation. Int. J. Robot. Res. 2017, 36, 414-435. [CrossRef]

32. Abdallah, I.B.; Bouteraa, Y.; Rekik, C. Design and development of 3d printed myoelectric robotic exoskeleton for hand rehabilitation. Int. J. Smart Sens. Intell. Syst. 2017, 10, 341-366. [CrossRef]

33. Bowers, M.; Goldfarb, N.; Jagetia, A.; Khajuriwala, R.; Kumar, A.; Lam, B.; Shah, N. Design of a Low Cost Robotic System to Aid in the Rehabilitation of Stroke Patients. 2017. Available online: https:/ /adjagetia. github.io/Documents / design-low-cost.pdf (accessed on 8 July 2019).

34. Anam, K.; Al-Jumaily, A.A. Active exoskeleton control systems: State of the art. Procedia Eng. 2012, 41, 988-994. [CrossRef] 
35. Noda, T.; Sugimoto, N.; Furukawa, J.; Sato, M.A.; Hyon, S.H.; Morimoto, J. Brain-controlled exoskeleton robot for BMI rehabilitation. In Proceedings of the 2012 12th IEEE-RAS International Conference on Humanoid Robots (Humanoids 2012), Osaka, Japan, 29 November-1 December 2012; pp. 21-27.

36. Li, Z.; Wang, B.; Sun, F.; Yang, C.; Xie, Q.; Zhang, W. sEMG-based joint force control for an upper-limb power-assist exoskeleton robot. IEEE J. Biomed. Health Inform. 2014, 18, 1043-1050. [PubMed]

37. Secciani, N.; Bianchi, M.; Meli, E.; Volpe, Y.; Ridolfi, A. A novel application of a surface ElectroMyoGraphy-based control strategy for a hand exoskeleton system: A single-case study. Int. J. Adv. Robot. Syst. 2019, 16, 1729881419828197. [CrossRef]

38. Li, Z.; Huang, Z.; He, W.; Su, C.Y. Adaptive impedance control for an upper limb robotic exoskeleton using biological signals. IEEE Trans. Ind. Electron. 2017, 64, 1664-1674. [CrossRef]

39. Yu, W.; Rosen, J.; Li, X. PID admittance control for an upper limb exoskeleton. In Proceedings of the 2011 American Control Conference, San Francisco, CA, USA, 29 June-1 July 2011; pp. 1124-1129.

40. Ryser, F.; Bützer, T.; Held, J.P.; Lambercy, O.; Gassert, R. Fully embedded myoelectric control for a wearable robotic hand orthosis. In Proceedings of the 2017 International Conference on Rehabilitation Robotics (ICORR), London, UK, 17-20 July 2017; pp. 615-621.

41. Ding, Y.; Galiana, I.; Siviy, C.; Panizzolo, F.A.; Walsh, C. IMU-based iterative control for hip extension assistance with a soft exosuit. In Proceedings of the 2016 IEEE International Conference on Robotics and Automation (ICRA), Stockholm, Sweeden, 16-21 May 2016; pp. 3501-3508.

42. Langtree, I. Height Chart of Men and Woman in Different Countries. In Canada: Disabled; 2018. Available online: https:/ / www.disabled-world.com/calculators-charts/height-chart.php (accessed on 1 December 2017).

43. Walpole, S.C.; Prieto-Merino, D.; Edwards, P.; Cleland, J.; Stevens, G.; Roberts, I. The weight of nations: An estimation of adult human biomass. BMC Public Health 2012, 12, 439. [CrossRef] [PubMed]

44. Legnani, G.; Palmieri, G. Fondamenti di Meccanica e Biomeccanica del Movimento; CittàStudi: Milano, Italy, 2016.

45. Lens, T.; Kirchhoff, J.; von Stryk, O. Dynamic modeling of elastic tendon actuators with tendon slackening. In Proceedings of the 2012 12th IEEE-RAS International Conference on Humanoid Robots (Humanoids 2012), Osaka, Japan, 29 November-1 December 2012; pp. 779-784.

46. Roveda, L.; Pallucca, G.; Pedrocchi, N.; Braghin, F.; Tosatti, L.M. Iterative learning procedure with reinforcement for high-accuracy force tracking in robotized tasks. IEEE Trans. Ind. Inform. 2017, 14, 1753-1763. [CrossRef]

47. Lewis, F.L.; Vrabie, D.; Syrmos, V.L. Optimal Control; John Wiley \& Sons: Hoboken, NJ, USA, 2012.

48. Tao, C.W.; Taur, J.S.; Chen, Y. Design of a parallel distributed fuzzy LQR controller for the twin rotor multi-input multi-output system. Fuzzy Sets Syst. 2010, 161, 2081-2103. [CrossRef]

49. Roveda, L.; Haghshenas, S.; Prini, A.; Dinon, T.; Pedrocchi, N.; Braghin, F.; Tosatti, L.M. Fuzzy impedance control for enhancing capabilities of humans in onerous tasks execution. In Proceedings of the 2018 15th International Conference on Ubiquitous Robots (UR), Honolulu, HI, USA, 26-30 June 2018; pp. $406-411$.

50. Drive for Real-World Robots. Available online: https:/ / www.anybotics.com/anydrive-robotic-actuator / (accessed on 30 July 2019).

51. EUROBENCH. Available online: http:/ / eurobench2020.eu/ (accessed on 30 July 2019).

(C) 2019 by the authors. Licensee MDPI, Basel, Switzerland. This article is an open access article distributed under the terms and conditions of the Creative Commons Attribution (CC BY) license (http://creativecommons.org/licenses/by/4.0/). 